\title{
TUC338 promotes cell migration and invasion by targeting TIMP1 in cervical cancer
}

\author{
QIN LI ${ }^{1}$, FEIYANG SHEN ${ }^{2}$ and CHENGHAI WANG ${ }^{3-5}$ \\ ${ }^{1}$ Department of Clinic, School of Medicine, Yangzhou Polytechnic College, Yangzhou, Jiangsu 225009; \\ ${ }^{2}$ School of Medicine, Yangzhou University; ${ }^{3}$ Department of Pathology, The Affiliated Hospital of Yangzhou University, \\ Yangzhou, Jiangsu 225000; ${ }^{4}$ Jiangsu Key Laboratory of Integrated Traditional Chinese and Western Medicine for \\ Prevention and Treatment of Senile Disease; ${ }^{5}$ Jiangsu Co-Innovation Center for Prevention and Control of Important \\ Animal Infectious Disease and Zoonoses, Yangzhou University, Yangzhou, Jiangsu 225001, P.R. China
}

Received August 9, 2015; Accepted January 26, 2017

DOI: $10.3892 / \mathrm{ol} .2017 .5971$

\begin{abstract}
Ultraconserved regions (UCRs) are non-proteincoding gene sequences that are strictly conserved across numerous distinct species. It has been demonstrated previously that UCRs encoding non-coding RNAs serve as regulators of gene expression. In recent decades, there has been increasing evidence for the involvement of UCRs in carcinogenesis. In previous studies, the non-coding RNA transcribed ultraconserved element 338 (TUC338) was identified to serve an oncogenic role in hepatocellular cancer; however, thus far, the role of TUC338 in cervical cancer (CC) remains undefined. The results of the present study revealed that TUC338 is significantly upregulated in $\mathrm{CC}$ tissues and cell lines, and that the upregulation of TUC338 is associated with lymph node metastasis. Transfection with small interfering RNA (siRNA) against TUC338 could markedly inhibit cell migration and invasion in HeLa and C33A CC cell lines. Using a dual-luciferase reporter assay, tissue inhibitor of metalloproteinase 1 (TIMP1) was demonstrated to be negatively regulated by TUC338 at the post-transcriptional level, via a specific target site within the $3^{\prime}$ untranslated region. The expression of TIMP1 was also observed to be inversely associated with TUC338 expression in CC tissues. Overexpression of TIMP1 with MigRI-TIMP1-green fluorescent protein inhibited CC cell migration and invasion and downregulated matrix metalloproteinase 9, resembling the effects of TUC338 siRNA. Therefore, the results of the present study suggest that TUC338 acts as a novel oncogene by targeting the TIMP1 gene, and inhibiting CC cell migration and invasion.
\end{abstract}

Correspondence to: Dr Chenghai Wang, Department of Pathology, The Affiliated Hospital of Yangzhou University, 368 Hanjiangzhong Road, Yangzhou, Jiangsu 225000, P.R. China

E-mail: 429350063@qq.com

Key words: cervical cancer, transcribed ultraconserved element 338 , tissue inhibitor of metalloproteinase 1 , matrix metalloproteinase 9 , migration, invasion

\section{Introduction}

Cervical cancer (CC) is the second most common type of female-specific cancer, ranking only behind breast cancer, and the fourth leading cause of cancer-associated mortality in females worldwide (1). The majority of cases of CC, $~ 80 \%$, occur in developing countries (2). As detection of CC precursors by cytological examination and their eradication by laser vaporization or cone biopsy are the most effective methods of preventing $\mathrm{CC}$, the carcinoma is treatable, and, in many cases, curable. However, once cancer advances and spreads to the distant lymph nodes, the prognosis is poor (3). Therefore, it is essential to identify metastasis-associated molecules and to improve our understanding of the underlying molecular mechanisms of the metastasis of $\mathrm{CC}$.

Ultraconserved regions (UCRs) are non-coding gene sequences that are strictly conserved across mice, rats and humans. UCRs regulate the expression and translation of mRNAs. It has been demonstrated that UCRs encode non-coding RNAs (ncRNAs) that serve as modulators of gene expression $(4,5)$.

Previous genome expression profiling studies demonstrated that transcribed ultraconserved RNAs (TUC-RNAs) exhibit distinct profiles in human carcinomas $(6,7)$. Furthermore, certain TUC-RNAs are able to serve as oncogenes or tumor suppressor genes in tumor development. For example, transcribed ultraconserved element 73 (TUC73) has been demonstrated to regulate cell proliferation and apoptosis in colorectal cancer cell lines (6). However, the regulation of the majority of TUC-RNAs and their underlying molecular mechanisms remain unknown in $\mathrm{CC}$.

TUC338 is a TUC-RNA that serves as a tumor oncogene. Braconi et al (8) demonstrated that TUC338 is markedly overexpressed in hepatocellular cancer (HCC) cell lines and tissue, and it promotes tumor cellular proliferation and modulates progression through regulation of the cell cycle. However, the expression level and biological role of TUC338 in CC remains unclear.

In the present study, the differential expression of TUC338 in human CC samples was identified using reverse transcription-quantitative polymerase chain reaction (RT-qPCR), and the function of TUC338 in the migration and invasion of CC cells was investigated. In addition, to understand the 
underlying molecular mechanism of CC metastasis, the target gene of TUC338 was further investigated. To the best of our knowledge, the present study is the first to investigate the expression and mechanism of TUC338 in CC migration and invasion. The results of the present study identify TUC338 as a novel target for further therapeutic studies of CC.

\section{Materials and methods}

CC tissue collection. $\mathrm{CC}$ tissue specimens $(\mathrm{n}=40)$ and normal cervical tissues $(n=40)$ were obtained from female patients with CC (age range, 40-68 years) at the Department of Obstetrics and Gynecology of the Jiangsu Subei People's Hospital, affiliated to Yangzhou University (Yangzhou, China) from January 2015 to January 2016. At the time of sample collection, the patients were untreated, and the samples were taken during initial diagnosis as part of routine examination.

The absence of tumor cells in the matched normal tissues was confirmed by a pathologist. All tissues were obtained during surgery and immediately stored in liquid nitrogen prior to use. Approval for the present study was granted by the Institutional Medical Research Ethics Committee of the Medical College of Yangzhou University (Yangzhou, China). All patients provided written informed consent.

Cell line culture. Human CC cell lines (HeLa and C33A) were purchased from the Chinese Peking Union Medical College Cell Bank (Beijing, China). All cell lines were maintained in RPMI-1640 'medium (HyClone; GE Healthcare Life Sciences, Logan, UT, USA) supplemented with $10 \%$ fetal bovine serum (FBS; HyClone; GE Healthcare Life Sciences), 100 IU/ml penicillin and $100 \mathrm{mg} / \mathrm{ml}$ streptomycin (Beyotime Institute of Biotechnology, Haimen, China) at $37^{\circ} \mathrm{C}$ in a humidified atmosphere containing $5 \% \mathrm{CO}_{2}$.

RT-qPCR detection and quantification. Total RNA was isolated from cells using TRIzol ${ }^{\circledR}$ reagent (Invitrogen; Thermo Fisher Scientific, Inc., Waltham, MA, USA), according to the manufacturer's protocol. Purification of RNA (1 $\mu \mathrm{g})$ was performed using TRIzol ${ }^{\circledR}$ Plus RNA Purification kit (Thermo Fisher Scientific, Inc.). RNA was reverse-transcribed into cDNA using the PrimeScript First Strand cDNA Synthesis kit (Takara Bio, Inc., Otsu, Japan), according to the manufacturer's protocol. qPCR was performed using the UltraSYBR Mixture kit (CWBIO; Kang Wei Biological Technology Co. Ltd., Beijing, China) on an Applied Biosystems 7500 Real-Time PCR system (Applied Biosystems; Thermo Fisher Scientific, Inc.). The thermocycling conditions for $\mathrm{qPCR}$ were; pre-denaturation at $95^{\circ} \mathrm{C}$ for $10 \mathrm{~min}$, denaturing at $95^{\circ} \mathrm{C}$ for $15 \mathrm{sec}$ and annealing/extending at $60^{\circ} \mathrm{C}$ for $1 \mathrm{~min}$ for 40 cycles. U6 small nuclear ribonucleoprotein RNA and $\beta$-actin mRNA were used as internal controls. All reactions were run in triplicate using the following primers: TUC338 forward, 5'-GCAGCGACAGTGCGAGCT-3' and reverse, 5'-TCCGAG TGAGTTAGGAAG-3', U6 forward, 5'-CGCTTCGGCAGC ACATATAC-3' and reverse, 5'-TTCACGAATTTGCGTGTC AT-3', tissue inhibitor of metalloproteinase 1 (TIMP1) forward, 5'-CTTCTGGCATCCTGTTGT-3' and reverse, 5'-ACTGCA GGTAGTGATGTG-3', matrix metalloproteinase-9 (MMP9) forward, 5'-GTGATTGACGACGCCTTT-3' and reverse,
5'-CAACTCGTCATCGTCG-3' and $\beta$-actin forward, 5'-GTC ACCAACTGGGACGACAT-3' and reverse, 5'-GAGGCGTAC AGGGATAGCAC-3'. The $2^{-\triangle \Delta C q}$ method was used for quantification (9).

Transfection of CC cells. Full-length murine TIMP1 mRNA was cloned into the MigRI-green fluorescent protein (GFP) retroviral vector at the Noncoding RNA Center, Yangzhou University (Yangzhou, China) and the sequence was confirmed by Sangon Biotech Co., Ltd. (Shanghai, China). The vector was obtained from Dr Xiaoyun Dong (Temple university, PA, USA). Small interfering RNAs (siRNAs) against TUC338 were purchased from Santa Cruz Biotechnology, Inc. (Dallas, TX, USA). The siRNA sequences (5'-3') were as follows: siRNA-1, UGACAGCCCUGGAGACUGA; siRNA-2, CCACAGGAC AGGUACAGCA (8). HeLa and C33A cells were transfected with $60 \mathrm{nM}$ TUC338 siRNA (8) or $0 \mathrm{nM}$ siRNA as the negative control (NC) by Lipofectamine ${ }^{\circledR} 2000$ (Invitrogen Life Technologies; Thermo Fisher Scientific, Inc.) for $48 \mathrm{~h}$ prior to further experiments, according to the manufacturer's instructions. The transfected cells were incubated at $37^{\circ} \mathrm{C}$ with $5 \%$ $\mathrm{CO}_{2}$. The TUC338 and TIMP1 RNA levels in the transfected $\mathrm{CC}$ cells were quantified using RT-qPCR.

Nucleotide blast. In order to identify potential targets of TUC338, the nucleotide Basic Local Alignment Search Tool (blast.ncbi.nlm.nih.gov/Blast.cgi?PROGRAM=blastn\&PAGE_ TYTYPE=BlastSearch\&LINK_LOC=blasthome) was used. This provided complementary sequences of two genes TUC338 and TIMP1.

Dual-luciferase reporter assay. The full-length 3'-untranslated region (UTR) of TIMP1 was amplified by PCR from genomic DNA and cloned into the EcoRI and XhaI sites of a pGL3-BS vector (Promega Corporation, Madison, WI,USA). The primers for TIMP1 3'-UTR were as follows: forward, 5'-GTGAAT TCATCCTGCCCGGAGTGGAA-3' and reverse, 5'-GTTCTA GATTTCTGCTGGGTGGTAAC-3'. The mutant construct of TIMP1 3'-UTR was generated using a QuikChange ${ }^{\mathrm{TM}}$ mutagenesis kit (Stratagene; Agilent Technologies, Inc., Santa Clara, CA, USA). Co-transfection of reporter vectors and TUC338 siRNA or NC was performed using Lipofectamine ${ }^{\circledR} 2000$ (Invitrogen; Thermo Fisher Scientific, Inc.), according to the manufacturer's protocol. After $48 \mathrm{~h}$, dual-luciferase activity was determined using a Dual-Luciferase Reporter Assay System (Promega Corporation) according to the manufacturer's protocol.

Migration and invasion assay. In vitro cell migration and invasion assays were performed using Transwell chambers as described previously (10). For the migration assays, $5 \times 10^{4}$ cells (HeLa and C33A, transfected with TUC338 siRNA or NC) were added into the upper Transwell chamber (pore size, $8 \mu \mathrm{m}$; BD Biosciences, Franklin Lakes, NJ, USA). For the invasion assays, $1 \times 10^{5}$ cells (HeLa and C33A, transfected with TUC338 siRNA or NC) were added into the upper Transwell chamber (pore size, $8 \mu \mathrm{m}$ ) precoated with Matrigel (BD Biosciences). In both assays, cells were plated in medium without serum and medium containing $10 \%$ FBS in the lower chamber served as the chemoattractant. Following incubation $(14 \mathrm{~h})$ at $37^{\circ} \mathrm{C}$ in a $5 \% \mathrm{CO}_{2}$ humidified atmosphere, the cells that had not migrated 

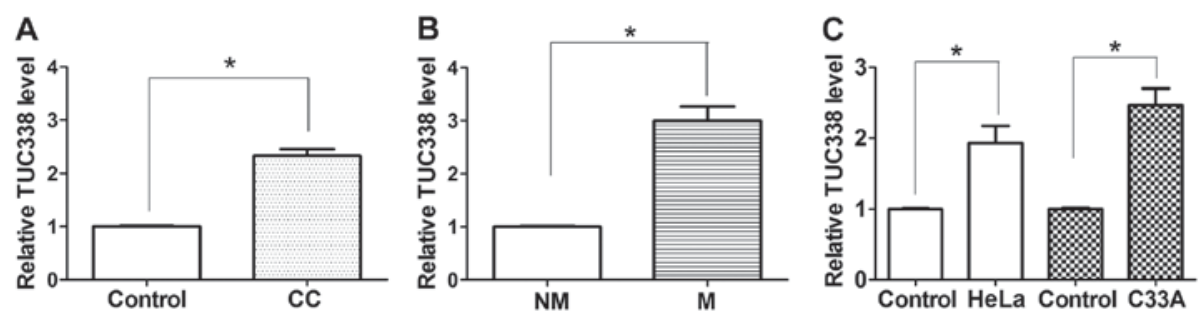

Figure 1. TUC338 is upregulated in cervical carcinoma. TUC338 expression levels were determined using reverse transcription-quantitative polymerase chain reaction and normalized against an endogenous U6 RNA control. (A) Expression levels of TUC338 in 40 paired human CC tissues relative to their corresponding normal cervical tissue samples (control). (B) Expression levels of TUC338 in patients with CC with lymph node metastasis (n=25) relative to non-metastatic samples (n=7). (C) Expression levels of TUC338 in two CC cell lines (HeLa and C33A) relative to human normal cervical tissue cells (control). Data are presented as the mean \pm standard deviation of three independent experiments per group. $\mathrm{P}<0.05$. TUC338, transcribed ultraconserved element 338 ; $\mathrm{CC}$, cervical cancer; $\mathrm{M}$, metastasis; NM, no metastasis.

or invaded through the pores were removed. The filters were fixed in $90 \%$ alcohol and stained with crystal violet $(0.1 \%$, $20 \mathrm{~min})$. A total of 5 random fields/chamber were counted using an inverted microscope (Olympus, Japan), and each test was performed in triplicate.

Western blot analysis. Proteins were extracted using cell lysis buffer for western and immunoprecipitation (Beyotime Institute of Biotechnology) according to the manufacturer's instructions. Protein concentration was quantified using an enhanced bicinchoninic acid assay kit (Beyotime Institute of Biotechnology), according to the manufacturer's protocol. For western blot analysis, equal amounts (5-10 $\mu \mathrm{g})$ of total protein were heated to $100^{\circ} \mathrm{C}$, and separated by $8.5 \%$ SDS-PAGE. Following electrophoresis, proteins were blotted onto a polyvinylidene difluoride membrane and blocked using bovine serum albumin for $2 \mathrm{~h}$ at room temperature. Membranes were incubated with anti-TIMP1 antibody (1:1,000; catalog no. 8946S; Cell Signaling Technology, Inc., Danvers, MA, USA), anti-MMP9 antibody (1:1,000; catalog no. 13667; Cell Signaling Technology, Inc.) or $\beta$-actin mouse monoclonal antibody (1:1,000; catalog no. cat-AF0003; Beyotime Institute of Biotechnology) overnight at $4^{\circ} \mathrm{C}$. TIMP1 protein level was detected using a horseradish peroxidase-conjugated anti-rabbit secondary immunoglobulin $\mathrm{G}$ antibody $(1: 1,000$; catalog no. A0208; Beyotime Institute of Biotechnology) for $2 \mathrm{~h}$ at room temperature. Protein bands were detected using a FluorChem FC2 Imaging system (ProteinSimple, San Jose, CA, USA).

Statistical analysis. Statistical analyses were performed using SPSS software (version 16.0; SPSS, Inc., Chicago, IL, USA). All plots were created using Microsoft Office Excel 2010 software (Microsoft Corporation, Redmond, WA, USA) and GraphPad Prism software (version 5; GraphPad Software, Inc., La Jolla, CA, USA). Results are expressed as the mean \pm standard deviation from three independent experiments. Differences were assessed using a two-tailed Student's t-test. The association between TIMP1 and TUC338 expression was tested using a two-tailed Pearson's correlation. $\mathrm{P}<0.05$ was considered to indicate a statistically significant difference.

\section{Results}

Expression of TUC338 is upregulated in CC tissues and cell lines. As TUC338 has been reported to be upregulated in HCC,
TUC338 expression levels were investigated in human CC tissues in the present study. The endogenous expression of TUC338 in human $\mathrm{CC}$ tissues and adjacent normal cervical tissues was determined using RT-qPCR. As presented in Fig. 1A, expression of TUC338 was upregulated in 92.5\% (37/40) of CC tissues compared with the corresponding adjacent normal cervical tissues. It was also identified that TUC338 expression was further upregulated in $92.59 \%$ (25/27) of patients with CC with lymph node metastasis compared with patients with $\mathrm{CC}$ without lymph node metastasis $(\mathrm{P}<0.05)$ (Fig. 1B). Similarly, the expression of TUC338 was observed to be significantly increased in two CC cell lines compared with normal human cervical tissue cells (Fig. 1C). These results suggest that increased TUC338 expression serves an important role in CC development and metastasis.

Downregulated expression of TUC338 via siRNA inhibits $C C$ cell migration and invasion. On the basis of the aforementioned results, it was investigated whether TUC338 served a role in regulating the migration and invasion of CC cells. HeLa and C33A cells were transfected with TUC338 siRNA or NC, and the transfection efficiency was evaluated using cell invasion and migration assays. As expected, transfection with TUC338 siRNAs significantly decreased TUC338 expression compared with the NC in HeLa and C33A cells (Fig. 2A and B). Furthermore, the cell migration and invasion assay demonstrated that downregulated expression of TUC338 resulted in a significantly decreased migration rate and invasion rate in HeLa and C33A cells compared with the NC (Fig. 2C and D). These results indicate that TUC338 acts as a tumor oncogene ncRNA and contributed to the promotion of migration and invasion of CC cells.

TIMP1 is a direct target of TUC338. ncRNAs are known to modulate hundreds of mRNA targets, resulting in global changes in the cell phenotype. In order to identify potential targets of TUC338, the nucleotide Basic Local Alignment Search Tool (blast.ncbi.nlm.nih.gov/Blast.cgi?PROGRAM=blastn\&PAGE_ TYPE=BlastSearch\&LINK_LOC=blasthome) was used. TIMP1 was identified as a putative target gene for TUC338 that mediates cell migration and invasion. To verify whether TUC338 targeted TIMP1 directly, a Dual-Luciferase reporter assay was performed. As presented in Fig. 3, in HeLa cells co-transfected with TIMP1-3'-UTR/pGL3-BS, transfection with TUC338 siRNA led to a significant decrease in luciferase activity compared with the group transfected with the NC 


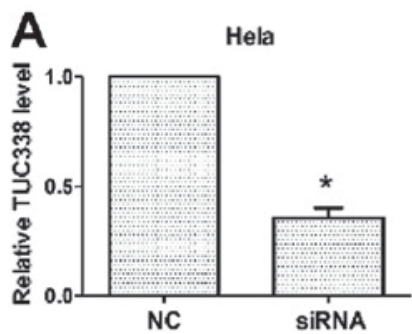

B C33A
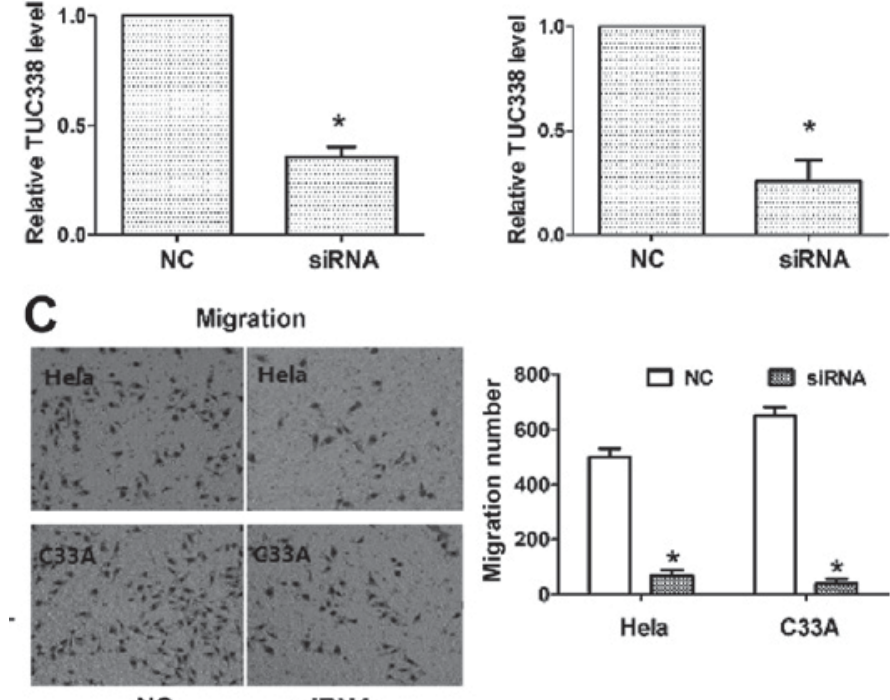

NC

SiRNA
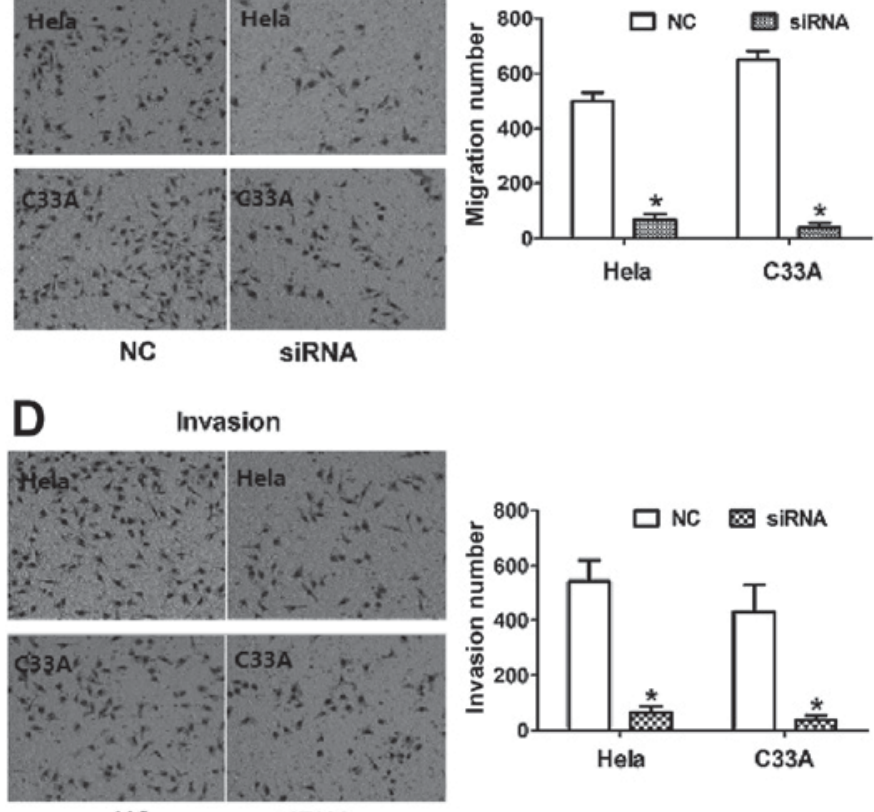

NC

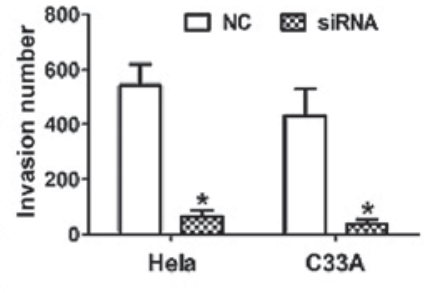

Figure 2. Knockdown of TUC338 inhibits migration and invasion of cervical cancer cells. Transfection of (A) HeLa and (B) C33A cells with TUC338 siRNA decreased the expression of TUC338, as determined using reverse transcription-quantitative polymerase chain reaction analysis, relative to cells transfected with NC. Transfection of HeLa and C33A cells with TUC338 siRNA also inhibited (C) migration and (D) invasion relative to the NC cells. "P<0.05 vs. NC. TUC338, transcribed ultraconserved element 338; siRNA, small interfering RNA; NC, negative control.

A

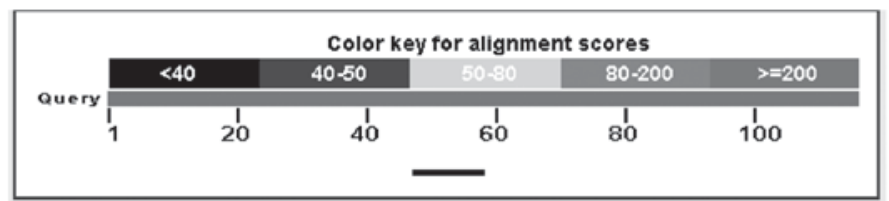

Sequence ID: Icl||Query_42299 Length: 618 Number of Matches: 1

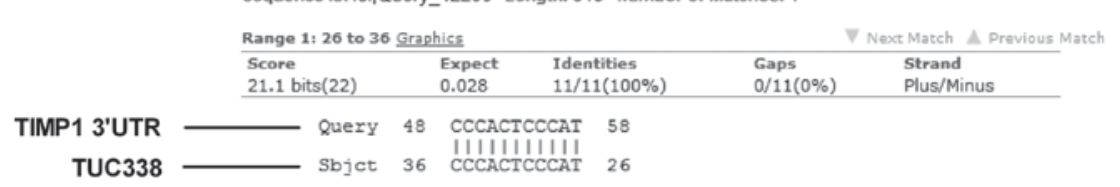

B

$\begin{array}{lllll}\text { 3' } & \text { CUCGgGUGAGGGUA } & 5^{\prime} & \text { TUC338 } \\ 5^{\prime} & \text { CAAGGGUGAGGGA } & 3^{\prime} & \text { wt TIMP1 } \\ \text { 5' } & \text { CAAG AUUGGGAGUA } & 3^{\prime} & \text { mut TIMP1 }\end{array}$

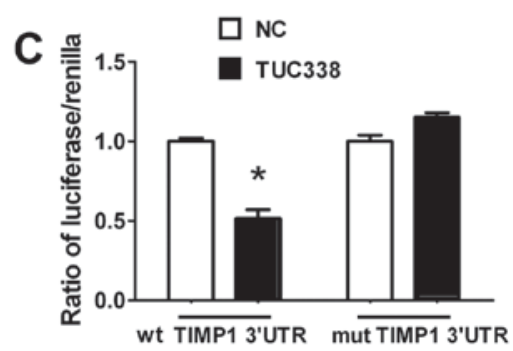

Figure 3. TIMP1 3'-UTR is a target of TUC338. (A) TIMP1 was identified as a target gene of TUC338 using Basic Local Alignment Search Tool prediction. (B) Schematic representations of the luciferase reporter plasmids with the wt or mut TIMP1 3'-UTR. (C) Luciferase activity in HeLa cells was determined following co-transfection with wt or mut TIMP1 3'-UTR and TUC338 siRNA relative to co-transfection with wt or mut TIMP1 3'-UTR and NC. "P<0.05 vs. NC. TIMP1, tissue inhibitor of metalloproteinase 1; UTR, untranslated region; TUC338, transcribed ultraconserved element 338; wt, wild-type; mut, mutant; LUC, luciferase; NC, negative control. 


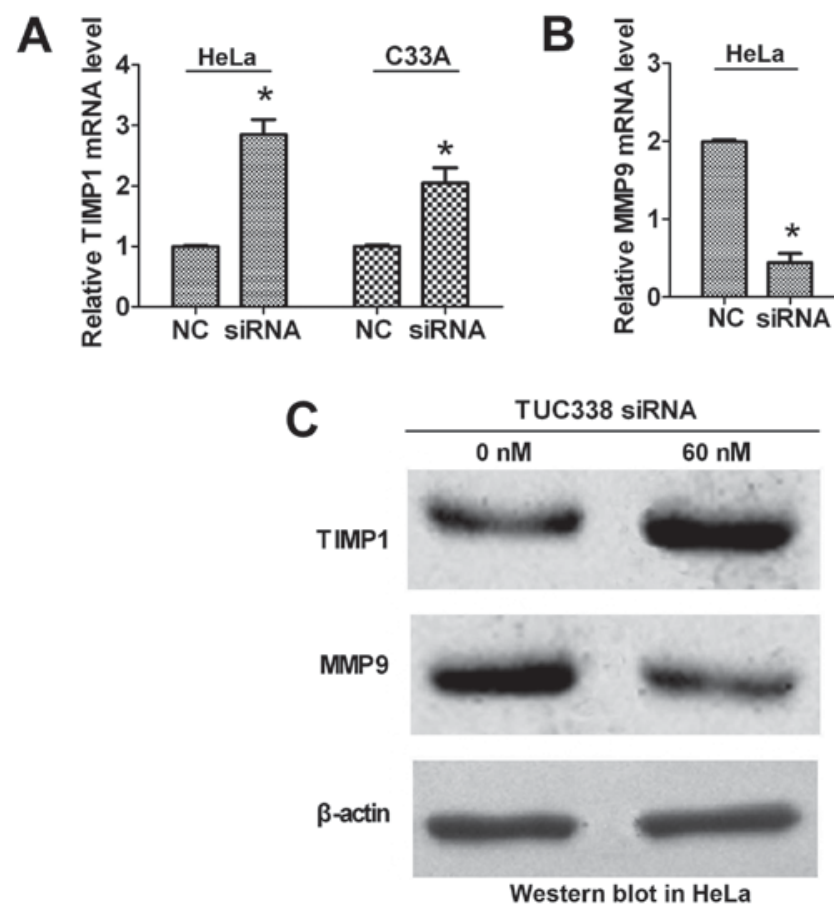

Figure 4. TUC338 negatively regulates TIMP1 expression and upregulates MMP9 gene expression in cervical cancer cells. (A) Expression of TIMP1 mRNA was significantly upregulated in HeLa and C33A cells following siRNA knockdown of TUC338 compared with NC. (B) Expression of MMP9 mRNA was significantly downregulated in HeLa and C33A cells following siRNA knockdown of TUC338 compared with NC. (C) Expression of TIMP1 protein was increased and expression of MMP9 protein was decreased in HeLa cells following siRNA knockdown of TUC338 compared with in the absence of TUC338 siRNA. $\beta$-actin was used as a loading control. ${ }^{*} \mathrm{P}<0.05$ vs. NC. TUC338, transcribed ultraconserved element 338; TIMP1, tissue inhibitor of metalloproteinase 1; MMP9, matrix metalloproteinase 9; siRNA, small interfering RNA; NC, negative control.
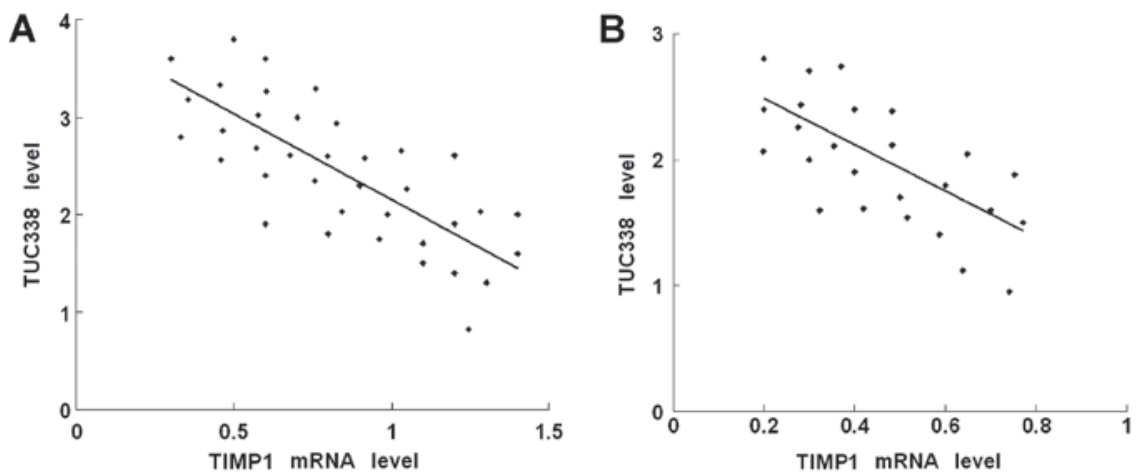

Figure 5. Expression of TUC338 and TIMP1 are inversely associated at the mRNA level. (A) Inverse association of TUC338 and TIMP1 expression in cervical carcinoma tissues without lymph node metastasis (two-tailed Pearson's correlation analysis, $\mathrm{n}=37, \mathrm{r}=-0.57, \mathrm{P}<0.05$ ). (B) Inverse association of TUC338 and TIMP1 expression in cervical carcinoma tissues with lymph node metastasis (two-tailed Pearson's correlation analysis, $\mathrm{n}=25, \mathrm{r}=-0.61, \mathrm{P}<0.05$ ). TUC338, transcribed ultraconserved element 338; TIMP1, tissue inhibitor of metalloproteinase 1.

$(\mathrm{P}<0.05)$. This repressive effect was eliminated by point mutations in the binding site of the TIMP1 3'-UTR. These results indicated that TUC338 exerts inhibitory effects on TIMP1 expression via interaction with the 3'-UTR of TIMP1.

TUC338 negatively regulates TIMP1 expression and upregulates matrix metalloproteinase 9 (MMP9) expression. To further confirm that TIMP1 is a target gene of TUC338 and that TIMP1 is a natural inhibitor of MMP9 (11), RT-qPCR and western blot analysis were performed to detect the expression of TIMP1 and MMP9 in association with TUC338 expression in HeLa and C33A cells. The results indicated that expression of TIMP1 was significantly upregulated and MMP9 was significantly downregulated at the mRNA level (Fig. 4A and B) following siRNA knockdown of TUC338 compared with the NC. At the protein level (Fig. 4C), TIMP1 was markedly upregulated and MMP9 was markedly downregulated following siRNA knockdown of TUC338 compared with NC. These results suggest that TUC338 negatively regulates the expression of its target gene TIMP1 at the post-transcriptional level, and that decreased expression of TIMP1 results in increased expression of MMP9.

Expression levels of TUC338 and TIMP1 are inversely correlated at the RNA level. To assess the association between TUC338 and TIMP1 in CC, the mRNA levels of TUC338 and TIMP1 were determined in CC and adjacent normal cervical tissues using RT-qPCR. As presented in Fig. 5A, when the TIMP1 mRNA expression levels were plotted against TUC338 
A

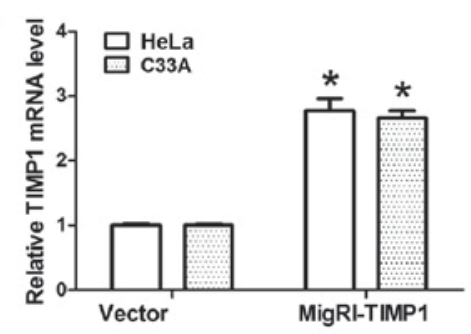

C
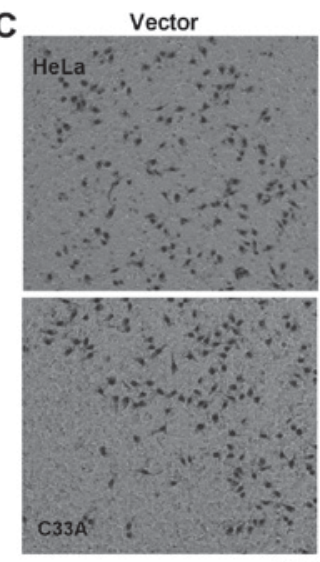

Migration

E

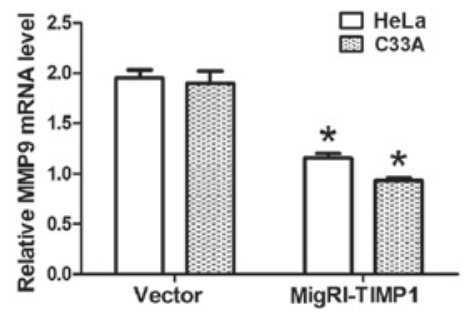

B
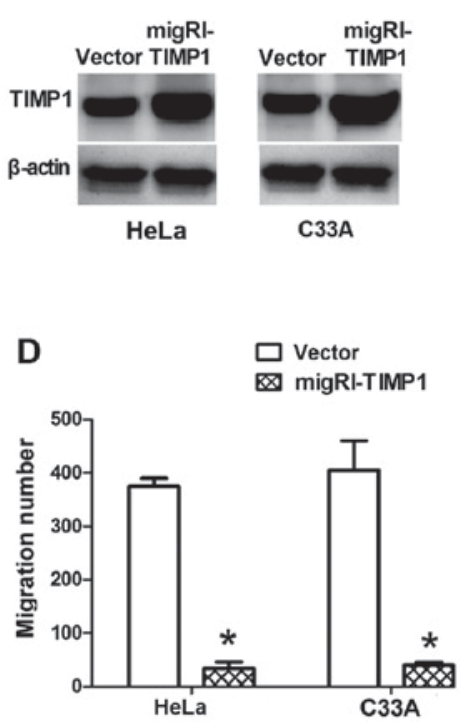

$\mathbf{F}$

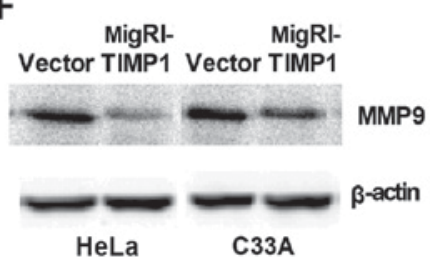

Figure 6. Overexpression of TIMP1 reduces migration of cervical cancer cells and downregulates MMP9. (A) TIMP1 mRNA levels were significantly upregulated in HeLa and C33A cells following transfection with MigRI-TIMP1-GFP relative to transfection with empty vector. (B) TIMP1 protein levels were markedly upregulated in HeLa and C33A cells following transfection with MigRI-TIMP1-GFP compared with transfection with empty vector. $\beta$-actin was used as a loading control. (C) Overexpression of TIMP1 markedly inhibited migration in cervical cancer cells compared with transfection with empty vector. (D) Quantification of migrating cells in HeLa and C33A cells. Overexpression of TIMP1 significantly decreased the number of migrating cells compared with transfection with empty vector. (E) Expression of MMP9 mRNA was significantly downregulated in HeLa and C33A cells following overexpression of TIMP1 compared with transfection with empty vector. (F) Expression of MMP9 protein was markedly downregulated in HeLa and C33A cells following overexpression of TIMP1 compared with transfection with empty vector. $\beta$-actin was used as a loading control. * P $<0.05$ vs. transfection with empty vector. TIMP1, tissue inhibitor of metalloproteinase 1; MMP9, matrix metalloproteinase 9; GFP, green fluorescent protein.

mRNA expression levels in up-regulated CC tissues, a significant inverse association was identified (two-tailed Pearson's correlation analysis, $\mathrm{n}=37, \mathrm{r}=-0.57 ; \mathrm{P}<0.05)$. Similarly, a significant inverse association was also identified in $\mathrm{CC}$ specimens with lymph node metastasis (Fig. 5B; two-tailed Pearson's correlation analysis, $\mathrm{n}=25, \mathrm{r}=-0.61 ; \mathrm{P}<0.05)$.

Overexpression of TIMP1 decreases the migration potential of $C C$ cells and upregulates MMP9. Expression of TIMP1 in CC cells was forced using transfection with MigRI-TIMP1-GFP plasmids. TIMP1 mRNA levels were significantly upregulated in HeLa and C33A cells following MigRI- TIMP1-GFP transfection (Fig. 6A); furthermore, TIMP1 protein levels were markedly upregulated in HeLa and C33A cells following MigRI-TIMP1-GFP transfection (Fig. 6B). Consistently, overexpression of TIMP1 markedly inhibited migration in CC cells (Fig. 6C and D), which resembled the inhibitory effects of TUC338 knockdown. It was also confirmed that the expression of MMP9 was significantly downregulated at the mRNA level (Fig. 6E) and at the protein level (Fig. 6F) following overexpression of TIMP1. These results suggested that TIMP1 modulates the migration of CC cells and acts as an inhibitor of MMP9 by negatively regulating its gene expression.

\section{Discussion}

UCRs are non-coding gene sequences that are strictly conserved across mice, rats and humans. UCRs regulate the expression and translation of mRNAs. By regulating protein production post-transcriptionally, a number of UCRs act as oncogenes or tumor suppressor genes $(6-8,12,13)$. However, the regulation of TUC338 and its underlying molecular mechanisms of action in CC are unknown.

The results of the present study indicated that TUC338 exhibited increased expression in CC tissues compared with in matched normal cervical tissues. The results also 
indicated that TUC338 siRNAs inhibited the invasion and migration of two CC cell lines. These results suggest that TUC338, as a novel tumor oncogene, serves a role in the metastasis and infiltration of CC. In addition, TIMP1 was identified as a potential target of TUC338 using theoretical prediction software. TIMP1 is a member of the TIMP family, which are natural inhibitors of the MMPs, a group of peptidases involved in degradation of the extracellular matrix. TIMP1 participates in the majority of the physiological and pathological processes involved in cell proliferation, migration, matrix remodeling, cell survival and matrix degradation (14-18). It has also been reported that TIMP1 is overexpressed in colorectal and lung tumors $(19,20)$, and is associated with metastasis and invasion in breast, upper urinary tract and oral squamous cell tumors (19-22).

In the present study, an inverse association between TUC338 and TIMP1 expression was identified in CC tissues. The results also demonstrated that TIMP1 was negatively regulated by TUC338 at the post-transcriptional level, via a specific target site within the 3'-UTR, and that TUC338 inhibited CC cell migration and invasion through the TIMP1-MMP9 signaling pathway. These findings suggest that a decrease in TIMP1 expression, induced by the suppression of TUC338, allows the progression of CC. Furthermore, this implicates dysregulation of the TIMP1 signaling pathway by UCRs as an important mechanism underlying cancer metastasis, particularly in cancer cell migration and cell invasion.

Metastasis is the movement of cancer cells from one organ or tissue to another. Its sequential events include detachment, migration, local invasion, formation of tumor embolus, extravasation and establishment in different organs $(23,24)$. Certain ncRNAs are able to regulate tumor metastasis signaling pathways (25). The identification of TUC338 as an important regulator of tumor cell migration and invasion in vitro emphasizes an essential role for this UCR in mediating $\mathrm{CC}$ oncogenesis and tumor behavior.

In summary, the results of the present study demonstrate that TUC338 is upregulated in CC, and that its upregulation is significantly associated with lymph node metastasis. Furthermore, siRNAs against TUC338 can inhibit the invasion and migration of CC cells. TUC338 can directly inhibit TIMP1 expression by targeting its 3'-UTR; TIMP1 was identified to be downregulated and inversely associated with TUC338 levels in CC. The results of the present study suggest that TUC338 is an important oncogene in $\mathrm{CC}$, and this knowledge may lead to novel treatments to prevent $\mathrm{CC}$ metastasis.

\section{Acknowledgements}

The present study was supported by Yangzhou Key Research Project-Social Development Plan (grant no. YZ2016065), 2016 High-level Talents Science Start-up Foundation in Yangzhou University (grant no. $2016 \mathrm{WCH}$ ) and the China National Science Foundation (grant no. 81273214).

\section{References}

1. Jemal A, Bray F, Center MM, Ferlay J, Ward E and Forman D: Global cancer statistics. CA Cancer J Clin 61: 69-90, 2011.

2. Kent A: HPV vaccination and testing. Rev Obstet Gynecol 3: 33-34, 2010.
3. Kyung MS, Kim HB, Seoung JY, Choi IY, Joo YS, Lee MY, Kang JB and Park YH: Tumor size and lymph node status determined by imaging are reliable factors for predicting advanced cervical cancer prognosis. Oncol Lett 9: 2218-2224, 2015.

4. Bejerano G, Lowe CB, Ahituv N, King B, Siepel A, Salama SR, Rubin EM, Kent WJ and Haussler D: A distal enhancer and an ultraconserved exon are derived from a novel retroposon. Nature 441: 87-90, 2006.

5. Katzman S, Kern AD, Bejerano G, Fewell G, Fulton L, Wilson RK, Salama SR and Haussler D: Human genome ultraconserved elements are ultraselected. Science 317: 915, 2007.

6. Calin GA, Liu CG, Ferracin M, Hyslop T, Spizzo R, Sevignani C, Fabbri M, Cimmino A, Lee EJ, Wojcik SE, et al: Ultraconserved regions encoding ncRNAs are altered in human leukemias and carcinomas. Cancer Cell 12: 215-229, 2007.

7. Lujambio A,Portela A,Liz J, Melo SA, Rossi S,Spizzo R, Croce CM Calin GA and Esteller M: CpG island hypermethylation-associated silencing of non-coding RNAs transcribed from ultraconserved regions in human cancer. Oncogene 29: 6390-6401, 2010.

8. Braconi C, Valeri N, Kogure T, Gasparini P, Huang N, Nuovo GJ, Terracciano L, Croce CM and Patel T: Expression and functional role of a transcribed noncoding RNA with an ultraconserved element in hepatocellular carcinoma. Proc Natl Acad Sci USA 108: 786-791, 2011.

9. Livak KJ and Schmittgen TD: Analysis of relative gene expression data using real-time quantitative PCR and the 2(-Delta Delta C(T)) method. Methods 25: 402-408, 2001.

10. Wang C, Yan G, Zhang Y, Jia X and Bu P: Long non-coding RNA MEG3 suppresses migration and invasion of thyroid carcinoma by targeting of Rac1. Neoplasma 62: 541-549, 2015.

11. Pietruszewska W, Bojanowska-Poźniak K and Kobos J: Matrix metalloproteinases MMP1, MMP2, MMP9 and their tissue inhibitors TIMP1, TIMP2, TIMP3 in head and neck cancer: An immunohistochemical study. Otolaryngol Pol 70: 32-43, 2016.

12. Mestdagh P, Fredlund E, Pattyn F, Rihani A, Van Maerken T, Vermeulen J, Kumps C, Menten B, De Preter K, Schramm A, et al: An integrative genomics screen uncovers ncRNA T-UCR functions in neuroblastoma tumours. Oncogene 29: 3583-3592, 2010.

13. Catucci I, Verderio P, Pizzamiglio S, Manoukian S, Peissel B Barile M, Tizzoni L, Bernard L, Ravagnani F, Galastri L, et al: SNPs in ultraconserved elements and familial breast cancer risk. Carcinogenesis 30: 544-546, 2009.

14. Ma J, Wang J, Fan W, Pu X, Zhang D, Fan C, Xiong L, Zhu H, $\mathrm{Xu}$ N, Chen R and Liu S: Upregulated TIMP-1 correlates with poor prognosis of laryngeal squamous cell carcinoma. Int J Clin Exp Pathol 7: 246-254, 2014.

15. Lorente L, Martín MM, López P, Ramos L, Blanquer J, Cáceres JJ, Solé-Violán J, Solera J, Cabrera J, Argueso M, et al: Association between serum tissue inhibitor of matrix metalloproteinase-1 levels and mortality in patients with severe brain trauma injury. PLoS One 9: e94370, 2014.

16. Ramer R, Fischer S, Haustein M, Manda K and Hinz B: Cannabinoids inhibit angiogenic capacities of endothelial cells via release of tissue inhibitor of matrix metalloproteinases-1 from lung cancer cells. Biochem Pharmacol 91: 202-216, 2014.

17. Niewiarowska K, Pryczynicz A, Dymicka-Piekarska V, Gryko M, Cepowicz D, Famulski W, Kemona A and Guzińska-Ustymowicz K: Diagnostic significance of TIMP-1 level in serum and its immunohistochemical expression in colorectal cancer patients. Pol J Pathol 65: 296-304, 2014.

18. Rojiani MV, Ghoshal-Gupta S, Kutiyanawalla A, Mathur S and Rojiani AM: TIMP-1 overexpression in lung carcinoma enhances tumor kinetics and angiogenesis in brain metastasis. J Neuropathol Exp Neurol 74: 293-304, 2015.

19. Ramos-DeSimone N, Hahn-Dantona E, Sipley J, Nagase H, French DL and Quigley JP: Activation of matrix metalloproteinase-9 (MMP-9) via a converging plasmin/stromelysin-1 cascade enhances tumor cell invasion. J Biol Chem 274: 13066-13076, 1999.

20. Morini M, Mottolese M, Ferrari N, Ghiorzo F, Buglioni S, Mortarini R, Noonan DM, Natali PG and Albini A: The alpha 3 beta 1 integrin is associated with mammary carcinoma cell metastasis, invasion, and gelatinase B (MMP-9) activity. Int J Cancer 87: 336-342, 2000.

21. Farina AR and Mackay AR: Gelatinase B/MMP-9 in tumour pathogenesis and progression. Cancers (Basel) 6: 240-296, 2014.

22. Groblewska M, Siewko M, Mroczko B and Szmitkowski M: The role of matrix metalloproteinases (MMPs) and their inhibitors (TIMPs) in the development of esophageal cancer. Folia Histochem Cy tobiol 50: 12-19, 2012.

23. Gupta GP and Massagué J: Cancer metastasis: Building a framework. Cell 127: 679-695, 2006

24. Klein CA: Cancer. The metastasis cascade. Science 321: 1785-1787, 2008.

25. Sreekumar R, Sayan BS, Mirnezami AH and Sayan AE: MicroRNA control of invasion and metastasis pathways. Front Genet 2: 58, 2011. 